\title{
Opioids for chronic noncancer pain: a new Canadian practice guideline
}

\author{
Andrea D. Furlan MD PhD, Rhoda Reardon Dip(P\&OT), Clarence Weppler BSc Pharm, for the \\ National Opioid Use Guideline Group (NOUGG)
}

$\infty \infty$

See related commentary by Chou, page 881

$\mathrm{U}$ ndertreated chronic noncancer pain and growing misuse of opioids are two challenges presented by opioid therapy. A new Canadian guideline addresses these challenges with recommendations and tools for safe and responsible selection, prescription, titration and monitoring of opioids. ${ }^{1}$

Chronic noncancer pain is a substantial public health problem in many societies, ${ }^{2-4}$ where it has immense negative impact both socially and economically. The most potent analgesics available, opioids have been shown to reduce pain from both nociceptive and neuropathic causes. ${ }^{5}$ Their efficacy in functional outcomes has been less obvious. Although some physicians are reluctant to prescribe opioids for chronic noncancer pain, Canadian prescribing trends of the past decade are disturbing. Canada's recorded prescription-opioid consumption increased by about 50\% between 2000 and 2004, ${ }^{6}$ a rate of increase greater than that of the United States during the same period. Canada is currently the world's third-largest per capita consumer of opioid analgesics. ${ }^{7}$ The increase in opioid prescribing has been accompanied by increases in misuse, abuse, serious injuries and overdose-related deaths among people taking these drugs. ${ }^{8}$

Canadian medical regulators (i.e., colleges of physicians and surgeons) have recognized a growing need for guidance in opioid use for chronic noncancer pain. In November 2007, they formed the National Opioid Use Guideline Group to collaborate in developing the Canadian guideline for safe and effective use of opioids for chronic noncancer pain. ${ }^{1}$ Their aim was to oversee the development and implementation of a guideline to assist physicians to manage patients with chronic noncancer pain by prescribing opioids in a safe and effective manner.

\section{Scope}

The Canadian guideline is intended to help physicians make appropriate decisions to start trials of opioid therapy for patients with chronic noncancer pain and to guide them in the monitoring of long-term opioid therapy, the detection of situations involving misuse, including addiction, and the appropriate response to such situations. The guideline was not designed to serve as a standard of care nor as a training manual. It addresses safe and effective prescribing of opioids for chronic noncancer pain (defined as pain that persists for more

\section{Key points}

- In patients with chronic noncancer pain, opioids may be effective and should be considered.

- Opioid therapy should begin with a setting of realistic goals with the patient, a monitored trial of dosage titration, and follow-up to ensure opioid effectiveness.

- Prescribers and dispensers can minimize potential harms associated with opioid use by assessing risks, educating patients, monitoring use over time, and reducing or stopping opioids when indicated.

- Good communication and collaboration between health care providers and patients, across clinical disciplines, and between primary care and specialty care, is important when treating patients with chronic noncancer pain.

than six months $)^{9}$ in male and female adolescents and adults, and is targeted to primary-care physicians, and medical and surgical specialists who manage patients with chronic noncancer pain. Pharmacists, nurses, and dentists may also find it useful. The scope of the guideline does not include use of opioids for acute pain or end-of-life pain, or treatment modalities and approaches for chronic noncancer pain other than opioids.

\section{Development}

\section{Leadership}

Three groups were instrumental in developing the Canadian guideline for safe and effective use of opioids for chronic noncancer pain. These groups were the National Opioid Use Guideline Group, a research group, and a national advisory panel. The role of the National Opioid Use Guideline Group was to oversee the development and implementation of the guideline; the group's members were appointed by medical regulatory bodies. The research group comprised a research librarian, a physician-epidemiologist and four physicianresearchers. This group was responsible for literature review,

From the Institute for Work and Health, Toronto Rehabilitation Institute, and the Department of Medicine, University of Toronto (Furlan); Quality Management Division, College of Physicians and Surgeons of Ontario (Reardon), Toronto, Ont.; and the Physician Prescribing Practices Department, College of Physicians \& Surgeons of Alberta (Weppler), Edmonton, Alta.

CMAJ 2010. DOI:10.1503/cmaj.100187 
quality appraisal, summary of the evidence, generation of the first draft of recommendations and revision of the recommendations using feedback from the national advisory panel.

The advisory panel consisted of 49 people invited to participate by the National Opioid Use Guideline Group. Selection of panel members was aimed at creating a well-balanced advisory body including multiple perspectives and varied experience. Selection was based on criteria that ensured crossCanada representation from among the target audience (i.e., family physicians and other physicians who manage chronic noncancer pain), other health care providers (e.g., pharmacists, nurses, psychologists) and patients with chronic noncancer pain. The role of the advisory panel was to comment and reach consensus on recommendations for practice. Identities of panel members were blinded to the research group and other panel members until the panel's last round of review.

Financial support for this work was provided by medical regulatory bodies; no funding was received from commercial organizations.

\section{Literature review}

For review of the literature, the research group relied on the meta-analysis reported in 2006 by Furlan and colleagues. ${ }^{5}$ Three new searches of the literature were also completed. The first targeted randomized controlled trials published since May 2006, with the goal of updating the Furlan meta-analysis. The second search targeted additional literature (i.e., studies of varying methodology) that addressed the treatment of chronic noncancer pain with opioids and managing the patient with problematic opioid use. The third search targeted additional literature (i.e., studies of varying methodology) that addressed long-term outcomes of opioid use.

In total, 6583 studies were identified; 184 (62 randomized controlled trials and 122 observational studies) met the criteria for inclusion and were used to develop recommendations. The methodologic quality of the randomized trials was assessed independently by two people using the Jadad instrument. ${ }^{10}$ The observational studies were not assessed for methodologic quality owing to lack of funding for experts in epidemiologic methods to perform the more complex and subjective reviews needed for observational studies.

During development of the Canadian guideline, additional searches of MEDLINE and www.guideline.gov were conducted for other guidelines on the same topic up to February 2009 , and 15 relevant guidelines were selected for detailed evaluation.

\begin{tabular}{|c|c|c|}
\hline Round & $\begin{array}{l}\text { Recommendations } \\
\text { under review, no. }\end{array}$ & Panelists participating, $\%$ \\
\hline 1 & 49 & 84 \\
\hline 2 & 20 & 80 \\
\hline 3 & 4 & 65 \\
\hline 4 & 2 & 60 \\
\hline
\end{tabular}

\section{Practice recommendations}

The research group provided methodologic and clinical expertise in the areas of chronic pain and addiction medicine. The group summarized evidence from the studies and drafted 49 initial recommendations that each included a discussion and related evidence. Final recommendations were produced using feedback from the national advisory panel through an iterative Modified Delphi process. ${ }^{11}$ Panelists received material via email and used an online survey tool to rate the relevance, feasibility and clarity of each recommendation, indicate their degree of agreement with each recommendation and provide open-ended comments.

Consensus was defined as support for a recommendation by $80 \%$ of panel respondents. Recommendations that did not receive this level of consensus were revised using feedback from the panel and rerated in the next round. With each round of review, panel members received a complete transcript of the written comments made by panelists in the previous round.

Although participation rates declined as the modified Delphi process progressed, the proportion of panel members involved remained high throughout (Table 1). Some drop-off occurring in the last two rounds may have been because of panel fatigue or related to the impact of the H1N1 influenza pandemic occurring in Canada at the time. Consensus on recommendations resulted after four rounds of electronic review and rating, culminating in a final meeting by telephone and web in which all panel members were invited to participate.

We adapted the system for grading levels of evidence developed by the Canadian Task Force on Preventive Health Care $^{12}$ (Table 2) and used this adapted system to grade the recommendations of the Canadian guideline, which are presented in Table 3. Details of the method can be found in the guideline (http://nationalpaincentre.mcmaster.ca/opioid/).

\section{Highlights}

The 24 practice recommendations of the Canadian guideline, organized in five clusters, are listed in Table 3, along with a summary of how the guideline assists practitioners in implementing the recommendations. An example based on Recommendation 9: Optimal dose is presented in Appendices 1 and 2 (available at www.cmaj.ca/cgi/content/full/cmaj.100187/DC1).

The Canadian guideline addresses four clinical questions commonly considered by physicians when deciding to use opioids to treat chronic noncancer pain:

- What should I do before writing a prescription for opioids?

- How do I titrate the dosage of opioids?

- What should I do to ensure patient safety?

- When do I stop a patient's opioid therapy?

The Canadian guideline includes numerous clinical tools (see http://nationalpaincentre.mcmaster.ca/opioid/).

\section{What should I do before writing a prescription for opioids?}

- Complete a thorough assessment to understand the nature of the pain and make an informed decision about whether opioids are a reasonable choice of treatment.

- Consider whether screening tools are needed to help identify whether the patient is at risk for opioid misuse or addiction. 
- Manage the patient's expectations by setting goals with the patient for functional improvement and pain reduction; these goals will be the outcomes used for measuring the opioid effectiveness.

- Ensure informed consent by reviewing potential benefits, risks, adverse effects and complications of opioid therapy with the patient .

\section{How do I titrate the opioid dosage?}

- Start with a low dosage, increase the dose gradually and monitor opioid effectiveness (i.e., an improvement in function or a reduction in pain intensity of at least 30\%).

- Track the daily dose in morphine equivalence and monitor for the "watchful dose" (i.e., $200 \mathrm{mg}$ morphine or equivalent per day). Therapy for most patients can be managed effectively below this amount. If you determine that the dosage required is beyond the watchful dose, reassess the pain to ensure opioids are the right therapy, reassess the risk for misuse and increase level of monitoring.

- Recognize that the optimal dose has been reached when three factors are in balance: the opioid is effective (i.e., the patient reports improved function or a reduction of at least $30 \%$ in pain intensity), effectiveness has reached a plateau (i.e., increasing the dosage yields negligible analgesic benefit), and no major adverse effects or complications are experienced.

\section{What should I do to ensure patient safety?}

- Use the goals for functional improvement and pain reduction that were set with the patient to monitor whether opioids are effective. Structured assessment tools may also be useful.

- Watch for aberrant drug-related behaviours that could signal opioid misuse. Tools available in the guideline can help with this.

- Assess factors that could impair the patient's cognition and psychomotor ability, which could make driving unsafe.

- Use available consultation as needed (e.g., when pain is unresponsive, when opioid misuse or addiction is suspected), or in special populations (e.g., patients who are pregnant, elderly or adolescent, or who have comorbid psychiatric conditions).

- Collaborate with pharmacists to improve patient education and safety.

\section{When do I stop a patient's opioid therapy?}

- Stop or switch opioids when adverse effects or risks are unacceptable or opioid effectiveness is insufficient.

- Discontinue opioids with a tapering dose protocol; avoid sedative-hypnotic drugs, especially benzodiazepines, during the taper.

\section{Implementation}

A national faculty was created to assist with implementation of the Canadian guideline. Thirty-five people from across the country were invited using criteria to ensure a mix of expertise and experience: educators (physician, interprofessional or patient education); those with a clinical focus or interest in topic; those connected to national or provincial knowledgetransfer infrastructure; or those perceived as a useful ambassador for the guideline.

The goals of the national faculty, as identified by participants, were to define outcomes of the implementation of the Canadian guideline and to develop plans to assist in implementation and evaluation. Activities initiated by the national faculty to ensure the Canadian guideline improves practice and patient outcomes will continue into the future, and coordination will be assumed by the Michael G. DeGroote National Pain Centre at McMaster University.

\section{Updating}

The Michael G. DeGroote National Pain Centre has accepted responsibility for stewardship of the Canadian guideline. This responsibility will include activities related to knowledge translation and updating the guideline by 2015 .

Table 2: Comparison of the system for grading of levels of evidence of the Canadian Task Force on Preventive Health Care (CTFPHC) with the adapted system used to grade recommendations of the Canadian guideline for safe and effective use of opioids for chronic noncancer pain

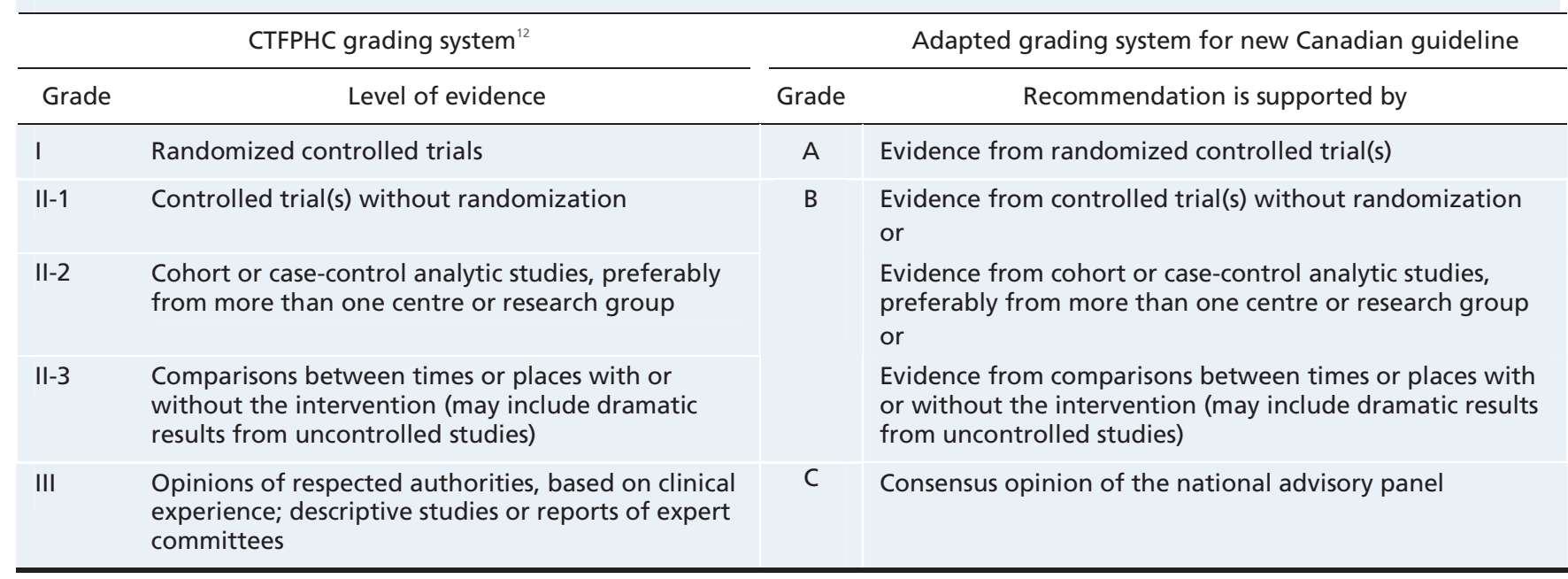


Table 3: Recommendations of the Canadian guideline for safe and effective use of opioids for chronic noncancer pain (1 of 3)

\begin{tabular}{l} 
Recommendation \\
\hline Deciding to initiate opioid therapy \\
1 Comprehensive assessment \\
Before initiating opioid therapy, ensure comprehensive \\
documentation of the patient's pain condition (Grade \\
C), general medical condition and psychosocial history \\
(Grade C), psychiatric status (Grade B), and substance \\
use history (Grade B).
\end{tabular}

B, C - A description of components of a comprehensive assessment

- Guides to interviews on alcohol consumption and substance use

- The CAGE questionnaire

Before initiating opioid therapy, consider using a screening tool to determine the patient's risk for opioid addiction.

\section{Urine screening for drugs}

When using urine drug screening to establish a baseline measure of risk or to monitor compliance, be aware of benefits and limitations, appropriate test ordering and interpretation, and have a plan to use results.

B

- An example of a screening tool (i.e., the Opioid Risk Tool)

C - Advice on patient education about urine screening for drugs, sample collection and tampering

- Comparison of point-of-care versus laboratory urine testing

- A list of the pros and cons of routine urine screening for drugs

- A table of information about interpreting and acting on unexpected results

- A table comparing immunoassay and chromatography, including detection times

A - Summaries of findings from randomized controlled trials

Before initiating opioid therapy, consider the evidence related to effectiveness in patients with chronic noncancer pain.

\section{$5 \quad$ Risks, adverse effects and complications}

Before initiating opioid therapy, ensure informed consent by explaining potential benefits, adverse effects, complications and risks (Grade B).

A treatment agreement may be helpful, particularly for patients not well known to the physician or at higher risk for opioid misuse (Grade C).

\section{Benzodiazepine tapering}

For patients taking benzodiazepines, particularly for elderly patients, consider a trial of tapering (Grade B). If a trial of tapering is not indicated or is unsuccessful, opioids should be titrated more slowly and at lower doses (Grade C).

\section{Conducting a trial of opioid therapy}

\section{Titration and driving}

During dosage titration in a trial of opioid therapy, advise the patient to avoid driving a motor vehicle until a stable dosage is established and it is certain the opioid does not cause sedation (Grade C), and when taking opioids with alcohol, benzodiazepines or other sedating drugs (Grade B).

\section{Stepped selection of an opioid}

During an opioid trial, select the most appropriate opioid for trial therapy using a stepped approach, and consider safety.
- Examples of conditions related to chronic noncancer pain for which opioids were shown to be effective in placebo-controlled trials and examples of conditions that have not been studied in controlled trials

B, C - A summary of potential risks, benefits, adverse effects and complications of opioid therapy

- Information about actions by physicians and education of patients and families aimed at reducing risks of overdose, diversion, addiction and withdrawal

- A patient-information handout that can be customized to a physician's practice

- An example of a treatment agreement, with suggestions about when such an agreement might be useful

B, C - Protocol for tapering benzodiazepines

- A table of benzodiazepine equivalence

B, C - Advice about conditions under which driving should be avoided during titration

- A definition of "pharmacologically stable dose"

C - Guidance for selecting the most appropriate opioid while considering severity of pain and patient-safety issues 
Table 3: Recommendations of the Canadian guideline for safe and effective use of opioids for chronic noncancer pain (2 of 3 )

\begin{tabular}{|c|c|}
\hline \multicolumn{2}{|c|}{ Recommendation } \\
\hline \multirow[t]{2}{*}{9} & Optimal dose \\
\hline & $\begin{array}{l}\text { When conducting a trial of opioid therapy, start with } \\
\text { a low dose, increase dosage gradually and monitor } \\
\text { analgesic effectiveness until optimal dose is attained. }\end{array}$ \\
\hline \multirow[t]{2}{*}{10} & Watchful dose \\
\hline & $\begin{array}{l}\text { Chronic noncancer pain can be managed effectively in } \\
\text { most patients with dosages at or below } 200 \mathrm{mg} / \mathrm{d} \text { of } \\
\text { morphine or equivalent (Grade A). Consideration of a } \\
\text { higher dosage requires careful reassessment of the } \\
\text { pain and of risk for misuse, and frequent monitoring } \\
\text { with evidence of improved patient outcomes (Grade } \\
\text { C). }\end{array}$ \\
\hline
\end{tabular}

$11 \quad$ Risk of misuse

When initiating a trial of opioid therapy for patients at higher risk for misuse, prescribe only for welldefined somatic or neuropathic pain conditions (Grade A), start with lower doses and titrate in smalldose increments (Grade B), and monitor closely for signs of aberrant drug-related behaviors (Grade C).

\section{Monitoring long-term opioid therapy}

When monitoring a patient on long-term therapy, ask about and observe for opioid effectiveness, adverse effects or medical complications, and aberrant drugrelated behaviours.

C - A definition of "optimal dose"

- A table showing suggested initial dosage and titration

- An example of how to assess change in pain intensity

A, C - Advice about actions to take when the morphineequivalent dosage approaches or exceeds $200 \mathrm{mg} / \mathrm{d}$

A, B, C - A description of patients at higher risk for misuse

- A tool for detecting aberrant drug-related behaviours

- Guidance on titration and monitoring in patients at higher risk for misuse

C - Information on elements of appropriate monitoring, including the value of physicianpharmacist collaboration

- An example of a tool to monitor functional improvement (i.e., the Brief Pain Inventory)

- Examples of tools for monitoring patients for aberrant drug-related behaviours

- An example of an opioid therapy record

B

- Guidance on initial doses when switching to a different opioid

For patients experiencing unacceptable adverse effects or insufficient opioid effectiveness from one particular opioid, try prescribing a different opioid or discontinuing therapy.

14 Driving and opioid therapy

When assessing safety to drive in patients on longterm opioid therapy, consider factors that could impair cognition and psychomotor ability, such as a consistently severe pain rating, disordered sleep and concomitant medications that increase sedation.

\section{Revisiting steps of trial therapy}

For patients receiving opioids for a prolonged period who may not have had an appropriate trial of therapy, take steps to ensure that long-term therapy is warranted and dose is optimal.

\section{Collaborative care}

When referring patients for consultation, communicate and clarify roles and expectations between primary-care physicians and consultants for continuity of care and for effective and safe use of opioids.
- Protocol for tapering opioids

- A conversion table for opioid analgesics

C - List of factors to assess that could impair cognition of "unsafe to drive" to a regulatory body

C - A checklist to assist physicians with patients taking long-term opioid therapy who have not been through the steps of a therapy trial, to ensure nothing has been missed

- An outline of the process for revisiting diagnosis, screening, patient education, selection and dosage of opioid and ensuring opioid effectiveness

C - Suggestions aimed at referring family physicians and consulting physicians on appropriate communication to ensure continuity of safe and effective care and psychomotor ability and thus prompt a report 
Table 3: Recommendations of the Canadian guideline for safe and effective use of opioids for chronic noncancer pain (3 of 3)

\begin{tabular}{ll}
\hline Recommendation \\
\hline Treating specific populations with long-term opioid the \\
$17 \quad$ Elderly patients \\
Opioid therapy for elderly patients can be safe and \\
effective (Grade B) with appropriate precautions \\
(Grade C), including lower starting doses, slower \\
titration, longer dosing interval, more frequent \\
monitoring, and tapering of benzodiazepines.
\end{tabular}

\section{Adolescent patients}

Opioids present hazards for adolescents (Grade B). A trial of opioid therapy may be considered for adolescent patients with well-defined somatic or neuropathic pain conditions when non-opioid alternatives have failed, risk of opioid misuse is assessed as low, close monitoring is available and consultation, if feasible, is included in the treatment plan (Grade C).

\section{Pregnant patients}

Pregnant patients taking long-term opioid therapy should be tapered to the lowest effective dose slowly enough to avoid withdrawal symptoms, and then therapy should be discontinued if possible.

\section{Comorbid psychiatric diagnosis}

Patients with a psychiatric diagnosis are at greater risk for adverse effects from opioid treatment. Usually in these patients, opioids should be reserved for welldefined somatic or neuropathic pain conditions. Titrate more slowly and monitor closely; seek consultation where feasible.

Grade(s) Tools available in guideline

$B$,

B, C - An overview of risks, risk reduction and prescribing-related cautions for elderly patients

- Protocol for tapering benzodiazepines

B, C - An overview of opioid use by and prescribing cautions for adolescent patients

B

B
- A list of cautions related to delivery and postpartum

- An overview of increased risks and prescribing cautions for patients with a psychiatric disorder

Managing opioid misuse and addiction in patients with chronic pain

21 Options for addiction treatment

For patients with chronic noncancer pain who are addicted to opioids, three treatment options should be considered: methadone or buprenorphine treatment (Grade A), structured opioid therapy (Grade B) or abstinence-based treatment (Grade C). Consultation or shared care, where available, can assist in selecting and implementing the best treatment option (Grade C).

\section{Prescription fraud}

To reduce prescription fraud, physicians should take precautions when issuing prescriptions and work collaboratively with pharmacists.

\section{Unacceptable behaviour by patients}

Be prepared with an approach for dealing with patients who disagree with their opioid prescription or exhibit unacceptable behaviour.

\section{Acute care prescription of opioids}

Acute or urgent health care facilities should develop policies to provide guidance on prescribing opioids for chronic pain to avoid contributing to opioid misuse or diversion.

\begin{abstract}
A, B, C - Indications for three treatment options for the opioid-addicted patient with chronic pain (i.e., methadone or buprenorphine treatment, structured opioid therapy, and abstinence-based treatment).
\end{abstract}

C A list of precautions that could reduce prescription fraud

C - A description of common sources of conflict between patients and physicians and how physicians can minimize these

- Advice on circumstances under which police should be contacted

C - Guidance for physicians in acute or urgent health care facilities on creating a policy on prescription of opioids

\section{Other guidelines}

Numerous other clinical practice guidelines address the management of chronic noncancer pain with opioids. A literature search showed that most other guidelines were either focused on a specific health problem (e.g., fibromyalgia, neuropathic pain, osteoarthritis, low-back pain) or were out of date.

Three current guidelines are similar to the Canadian guideline in scope, population, development, sponsorship, 
recommendations and presentation. When work began on the Canadian guideline, only one of these three, the guideline of the American Society of Interventional Pain Physicians, had been published (originally in $2006^{13}$ and updated in 2008). ${ }^{14}$ However, its target audience was interventional pain specialists. In 2009, when development of the Canadian guideline was well underway, the two other similar guidelines were published. The guideline of the American Pain Society and American Academy of Pain Medicine ${ }^{15}$ contains additional recommendations (not included in the Canadian guideline) on treatment of breakthrough pain, management of adverse effects, selection of short-acting versus long-acting preparations, special issues related to methadone, and awareness of state laws. The guideline of the Utah Department of Health ${ }^{16}$ is a compilation of recommendations from six other guidelines for the management of chronic noncancer pain with opioids. No major differences exist between the Utah and the Canadian guideline.

\section{Limitations}

The Canadian guideline is constrained by the paucity of supporting evidence in most of the topic areas in which recommendations for practice were considered necessary and relevant. This constraint necessitated a heavy reliance on the opinions and expertise of national advisory panelists for developing recommendations. Our literature searches for observational studies used broad terms and might have missed relevant studies. Of the 183 studies used to support the recommendations, only 62 were randomized trials, and the remainder were observational studies. Given that the quality of the observational studies was not formally assessed, we used an adaptation of the grading system of the Canadian Task Force on Preventive Health Care. Another limitation of the published evidence was that functional outcomes studied were predominantly activities of daily living and quality of life. Other important outcomes, such as return to work, productivity and cognitive impairment, were rarely reported.

Potential long-term complications of opioid use (e.g., hypogonadism, opioid-induced hyperalgesia, sleep apnea, addiction) may occur even if the Canadian guideline recommendations are strictly followed.

Although an attempt was made to maintain a nationwide perspective, the national advisory panel pointed out numerous instances in which draft recommendations were dependent on access to resources not available in all parts of Canada (e.g., pain or addiction specialists, multidisciplinary pain management teams, prescription monitoring databases).

The Canadian guideline addresses only one modality for managing chronic noncancer pain — opioid therapy — and it does not discuss or provide guidance on other options. In spite of its narrow focus, the Canadian guideline is a lengthy and detailed document, and will need to be reformatted into feasible and practical tools for day-to-day use by busy practitioners. Screening tools are only valid when the patient's reporting is accurate.

Finally, the National Opioid Use Guideline Group repre- sents medical regulatory authorities, which could create concern that the Canadian guideline will be used as a standard of practice rather than for its intended purpose as advice to assist physicians.

\section{Gaps in knowledge}

Questions remain that cannot be answered confidently by the currently published randomized trials and that require appropriately designed studies of long-term opioid use for chronic noncancer pain. Gaps in knowledge remain in areas involving:

- Alternative routes of administration. More information is needed on efficacy and risk-benefit ratios of intramuscular, subcutaneous, transdermal, rectal and infusion routes of administration of opioids for chronic noncancer pain.

- Comparison of opioids with non-opioid drugs. Welldesigned trials for equivalence and non-inferiority are needed to assess the relative effectiveness and risk-benefit ratios of opioids versus non-opioid drugs.

- Various clinical diagnoses. Most randomized trials on opioids for chronic noncancer pain concern musculoskeletal pain and neuropathic pain. Limited literature exists on treatment of fibromyalgia pain and chronic headache with opioids other than tramadol, and no useful literature exists on opioid treatment for chronic visceral pain.

- Long-term follow-up. Chronic noncancer pain is a longterm disorder, but the randomized controlled trials included in the current systematic review had fairly short follow-up periods. Well-designed long-term studies are needed to clarify the proportion of patients with chronic noncancer pain for whom opioids remain effective over months or years; the potential over extended time frames for opioid tolerance, hyperalgesia, loss of efficacy and complications such as hypogonadism, sexual dysfunction or central sleep apnea; and the probability of opioid misuse.

- Assessment of opioid misuse. Well-designed trials of sufficient duration are needed, with appropriate measures to identify prevalence and risks of opioid-related problems such as addiction.

- Populations with comorbidities. Trials are needed involving safe and appropriate management of chronic pain in patients with substantial comorbidity (e.g., pain in the elderly or in those with psychiatric comorbidity).

- Impact of research sponsorship. The majority of the randomized trials included in the systematic review were funded by the pharmaceutical industry. However, these studies did not include sufficient information to determine whether funding by the industry might have introduced publication bias. Whether small or unfavourable studies exist that were not submitted for publication is not known.

- The influence of genetic factors in opioid metabolism, analgesic response, incidence of adverse effects and predisposition to misuse and addiction. 


\section{This article has been peer reviewed.}

Competing interests: Andrea Furlan is a contracted member of the drug advisory committee of the Workplace Safety and Insurance Board (WSIB) of Ontario. She has been contracted by the Common Drug Review of the Canadian Agency for Drugs and Technology in Health to review an opioid medication, has received honoraria to write an expert opinion paper for the WSIB on opioid use for chronic noncancer pain, and has received grants from the Canadian Institutes of Health Research and the Ontario Alternative Funding Plan Innovation Fund to conduct research on opioids.

The institution(s) of Rhoda Reardon and Clarence Weppler received grants from the Federation of Medical Regulatory Authorities for this work. Rhoda Reardon is a full-time employee of the College of Physicians and Surgeons of Ontario, and Clarence Weppler is a full-time employee of the College of Physicians and Surgeons of Alberta. Clarence Weppler's wife is an employee of Servier Canada, and his daughter is an employee of GlaxoSmithKline.

Contributors: Andrea Furlan led the research group responsible for the systematic review and evidence synthesis, and contributed extensively to the manuscript. Rhoda Reardon and Clarence Weppler were co-chairs of the National Opioid Use Guideline Group (NOUGG), oversaw development of the guideline and coauthored the manuscript. All of the authors approved the final version of the manuscript submitted for publication.

Acknowledgements: Meldon Kahan and Angela Mailis-Gagnon led the development of the recommendations from initial draft, through revisions using feedback from the national advisory panel, to final copy. Emma Irvin designed and conducted the literature search, performed screening for relevance, and contributed methodological expertise on systematic reviews. Anita Srivastava and Luis Chaparro assisted with extraction of data and synthesis of evidence. Caroline Sutherland edited the guideline and the manuscript for this article. A full list of members of NOUGG is available at $\mathrm{http}: / /$ nationalpaincentre.mcmaster.ca/opioid/.

The National Opioid Use Guideline Group extends its sincere appreciation to members of the national advisory panel, whose contributions made the Canadian guideline possible, and the national faculty, for their continuing contribution in implementing the Canadian Guideline to practice.

Funding: NOUGG is grateful for support from the Federation of Medical Regulatory Authorities of Canada, the provincial and territorial medical regulatory authorities, and specifically acknowledges the significant contribution of funds and staff time from the College of Physicians and Surgeons of Ontario and the College of Physicians \& Surgeons of Alberta. The authors acknowledge and appreciate funding from CIHR that enabled members of our national faculty to meet in person. No funding was received from any other source.

\section{REFERENCES}

1. National Opioid Use Guideline Group. Canadian guideline for safe and effective use of opioids for chronic non-cancer pain. Hamilton (ON): NOUGG; 2010. Available: http://nationalpaincentre.mcmaster.ca/opioid/ (accessed 2010 May 3).

2. Ricci JA, Stewart WF, Chee E, et al. Back pain exacerbations and lost productive time costs in United States workers. Spine (Phila Pa 1976) 2006;31:3052-60.

3. Elliott AM, Smith BH, Penny KI, et al. The epidemiology of chronic pain in the community. Lancet 1999;354:1248-52.

4. Eriksen J, Ekholm O, Sjogren P, et al. Development of and recovery from longterm pain. A 6-year follow-up study of a cross-section of the adult Danish population. Pain 2004;108:154-62.

5. Furlan AD, Sandoval JA, Mailis-Gagnon A, et al. Opioids for chronic noncancer pain: a meta-analysis of effectiveness and side effects. CMAJ 2006;174:1589-94.

6. Drugs: estimated world requirements for 2007: statistics for 2005. New York (NY): International Narcotics Control Board; 2006.

7. Narcotic drugs: estimated world requirements for 2010: statistics for 2008. New York (NY): International Narcotics Control Board; 2009.

8. Kuehn BM. Opioid prescriptions soar: increase in legitimate use as well as abuse JAMA 2007;297:249-51.

9. College of Physicians and Surgeons of Ontario. Evidence-based recommendations for medical management of chronic non-malignant pain. Toronto $(\mathrm{ON})$ : The College; 2000 .
10. Jadad AR, Moore RA, Carroll D, et al. Assessing the quality of reports of randomized clinical trials: is blinding necessary? Control Clin Trials 1996;17:1-12.

11. Murphy MK, Black NA, Lamping DL, et al. Consensus development methods and their use in clinical guideline development. Health Technol Assessment 1998: 2(3).

12. Steven H. Woolf, Renaldo N. Battista, Geoffrey M. Anderson, et al and the Canadian Task Force on the Periodic Health Examination. J Cltn Epidemiol 1990;43:891-905.

13. Trescot AM, Boswell MV, Atluri SL, et al. Opioid guidelines in the management of chronic non-cancer pain. Pain Physician 2006;9:1-39.

14. Trescot AM, Helm S, Hansen $\mathrm{H}$, et al. Opioids in the management of chronic noncancer pain: an update of American Society of the Interventional Pain Physicians' (ASIPP) Guidelines. Pain Physician 2008;11(Suppl 2):S5-62.

15. Chou R, Fanciullo GJ, Fine PG, et al. Clinical guidelines for the use of chronic opioid therapy in chronic noncancer pain. J Pain 2009;10:113-30.

16. Utah Department of Health. Utah clinical guidelines on prescribing opioids for treatment of pain. Salt Lake City (UT): The Department; 2009.

Correspondence to: Clarence Weppler, College of Physicians \& Surgeons of Alberta, 2700, 10020-100 Street NW, Edmonton AB T5J 0N3; opioid.guideline@cpsa.ab.ca

\section{Canadian guideline for safe and effective use of opioids for} chronic non-cancer pain

http://nationalpaincentre.mcmaster.ca/opioid/

\section{In MDD, do you compromise tolerability for efficacy?}

\section{Or do you aim for both?}

*Major depressive disorder

\section{Wyeth}

(c) 2010 Wyeth Canada, owner, now a part of Pfizer Inc. 